\title{
REM sleep deprivation reduces emotionality in female rats
}

\author{
ROBERT A. HICKS, STEVEN GOMEZ, LINDA GONZALES, MAXINE KURODA, \\ NICOLAS J. ORME, and JOE REYES \\ San Jose State University, San Jose, California 95192
}

\begin{abstract}
Forty female Sprague-Dawley female rats were divided into control and REM sleep-deprived groups to measure the effects of REM deprivation on level of emotionality (i.e., frequency of defecation and urination during 15-min posttreatment open-field tests). We observed significantly lower levels of emotionality in the REM-deprived animals during both the immediate and the 21-day posttreatment tests.
\end{abstract}

Several studies have reported that REM sleep deprivation (RD) increased the level of open-field activity in rats. In two of these studies, Albert, Cicala, and Siegel (1970) and Ogilvie and Broughton (1976), this relationship was interpreted as indicative of a general relationship between $\mathrm{RD}$ and emotionality. Specifically, Albert et al. suggested that RD had increased emotionality, whereas Ogilvie and Broughton drew the opposite conclusion, which seems more likely to be valid. This is because, unlike Albert et al., Ogilvie and Broughton recorded the frequency of defecation and urination during the open-field test period and based their conclusion on the fact that frequency of defecation was significantly reduced in the RD animals.

In a more recent study, Hicks and Moore (1979) have provided data that clearly support the hypothesis that $\mathrm{RD}$ reduces level of emotionality in rats. That is, during a 15-min open-field test period, they observed significantly lower frequencies of defecation and urination in both of their RD groups.

A design difficulty in the aforementioned studies, which places a restriction on the generalizability of their results, is that in each case only male animals were used. Thus, a purpose of the present research was to replicate the Hicks and Moore (1979) study using female animals. A second and novel purpose of this study was to measure, roughly, the recovery gradient of emotionality subsequent to REM rebound.

\section{METHOD}

The animals were 40 Sprague-Dawley female rats that were 60 days old (weight range $=181-224 \mathrm{~g}$ ) at the time of the first test for emotionality. These animals were randomly assigned to one of four treatments: a dry confinement control (DC), a large-platform control (LP), a 2-day small-platform RD group

Address reprint requests to Robert A. Hicks, Department of Psychology, San Jose State University, San Jose, California 95192. This research was supported by Grant 1506 RR08192-01 awarded by the NIH Minority Biomedical Support Program.
(SP2), and a 4-day small-platform RD group (SP4), so that each group was made up of 10 animals.

$\mathrm{RD}$ was achieved using an adaptation of the so-called "flower-pot" technique (i.e., the technique used by Albert et al., 1970; Hicks \& Moore, 1979; Ogilvie \& Broughton, 1976). All the animals were housed in 18.9-liter buckets that were modified so that food was delivered ad lib from a feeder on the side of the bucket. The feeder was positioned to be within easy reach of the animals. The top of each bucket was covered with wire mesh. Drinking water was continuously available from a bottle that was attached to the top of the bucket, so that the spout was within easy reach of the animal. For the LP, SP2, and SP4 conditions, the buckets were filled with water $\left(19^{\circ} \mathrm{C}\right)$ to $1 \mathrm{~cm}$ from the surface of the platform. The water in the buckets was changed daily. Room temperature was a constant $22^{\circ} \mathrm{C}$. Throughout the study, these animals were maintained on a 12-h light-dark cycle, with lights on at $0700 \mathrm{~h}$.

All the animals were confined in the RD apparatus for a 4-day period; that is, the LP group spent 4 days on the large platform (diameter $=16.5 \mathrm{~cm}$ ), the SP2 group spent the first 2 days on the small platform (diameter $=6.5 \mathrm{~cm}$ ), the SP4 group spent 4 days on the small platform, and, finally, each member of the DC group spent 4 days in a dry RD apparatus with the large platform in place. Thus, all the animals experienced relatively equal exposure to the novel environment of the RD apparatus at the time of the first emotionality test.

Emotionality was measured by counting the frequency of defecation and urination that occurred during a 15 -min test period in an $81.3 \times 81.3 \times 38 \mathrm{~cm}$ open-field apparatus.

The animals were all 50 days old when they arrived from the supplier. Each animal was handled for a 10-min period each day for 6 days. Then the animals were weighed and randomly assigned to one of the four treatments. After a 4-day period of confinement, all the animals were towel dried and allowed a 10-min rest period before being tested in the open-field apparatus (which was thoroughly cleaned after each animal was run). After testing, each animal was weighed. All testing was done by two experimenters who were naive to the treatment condition under which each animal had been maintained. After the first posttreatment test, the animals were returned to individual cages, where they remained for 21 days. The procedure outlined above was then repeated for the 21-day posttreatment test.

\section{RESULTS AND DISCUSSION}

First, for each animal for each test, we computed an emotionality score by summing the frequency of defe- 
Table 1

Mean Emotionality Scores and Standard Deviations for RD and Control Animals for Each Posttreatment Test

\begin{tabular}{cccccr}
\hline & \multicolumn{4}{c}{ Treatment } \\
\cline { 2 - 3 } \cline { 5 - 6 } $\begin{array}{c}\text { Posttreatment } \\
\text { Test }\end{array}$ & Mean & SD & & Mean & SD \\
\cline { 2 - 3 } \cline { 5 - 6 } & & & \multicolumn{3}{c}{ RD } \\
\hline Immediate & 3.6 & 2.9 & .4 & .7 \\
21 Days & 3.3 & 2.4 & & 1.4 & 1.7 \\
\hline
\end{tabular}

Note-For the immediate test, the between-groups $t=4.85$ $(p<.001)$; for the 21-day test, the between-groups $t=2.76$ $(p<.01)$. For the control group, the between-tests $t=.36$ (n.s.); for the $R D$ group, the between-tests $t=2.56(p<.02)$.

cation and urination. Then, using these scores, we compared the two control groups and the two $\mathrm{RD}$ groups on both tests. The differences between the mean emotionality scores of the DC and LC groups for both the immediate and 21-day posttreatment tests were not significant $(\mathrm{t}=.23$ and $\mathrm{t}=.36$, respectively). This was also true for the SP2-SP4 comparisons $(t=1.33$ and $\mathrm{t}=-1.17$, respectively). Thus, the data from the DC and LC groups were combined to form a single RD group. The means and standard deviations for both of these groups for both of the posttreatment tests are listed in Table 1 .

As is shown in Table 1, the differences between the emotionality means of the control and RD groups for both the immediate and 21-day posttreatment tests were significant. Thus, these data, like those provided by Hicks and Moore (1979) and Ogilvie and Broughton (1976) for male animals, demonstrate that RD substantially reduces level of emotionality. Further, these data suggest that, to a lesser degree, emotionality remains suppressed in the RD group well beyond the period that is required for REM rebound. In this regard, it may be the case that there is an emotionality recovery gradient that requires a period of time greater than the 21-day period employed in this study. For, as is shown in Table 1, compared with the controls, the RD animals exhibited significantly less emotionality during the 21-day posttreatment test. However, the animals in the RD group exhibited significantly more emotionality during this test than they had during the immediate posttreatment test. As we have discussed elsewhere (Hicks, Hirshfield, Humphrey, Lauber, Giampaoli, \& Hawkins, 1981), the reasons for the effects of RD on these behaviors and the persistence of certain $\mathrm{RD}$ behavioral effects beyond REM rebound remain obscure and present a challenge for subsequent research.

\section{REFERENCES}

Albert, I., Cicala, G. A., \& Siegel, J. The behavior effects of REM sleep deprivation in rats. Psychophysiology, 1970, 6, $550-560$.

Hicks, R. A., Hirshfield, C., Humphrey, V., Lauber, A., Giampaoli, J., \& Hawkins, J. REM sleep deprivation and food competition in male rats. Physiology \& Behavior, 1981, 26, 245-247.

Hicks, R. A., \& Moore, J. A. REM sleep deprivation diminishes fear in rats. Physiology \& Behavior, 1979, 22, 689-692.

Ogilvie, R. D., \& Broughton, R. J. Sleep deprivation and measures of emotionality in rats. Psychophysiology, 1976, 13, 249-260.

(Received for publication May 1, 1981.) 